\title{
Determination of chiral condensate from low-lying eigenmodes of Mobius domain-wall Dirac operator
}

\section{G. Cossu}

High Energy Accelerator Research Organization (KEK), Tsukuba 305-0801, Japan

\section{H. Fukaya}

Department of Physics, Osaka University, Toyonaka 560-0043, Japan

\section{S. Hashimoto*}

High Energy Accelerator Research Organization (KEK), Tsukuba 305-0801, Japan and School of High Energy Accelerator Science, SOKENDAI (The Graduate University for Advanced Studies), Tsukuba 305-0801, Japan

E-mail: shoji.hashimoto@kek.jp

\section{T. Kaneko}

High Energy Accelerator Research Organization (KEK), Tsukuba 305-0801, Japan and SOKENDAI (The Graduate University for Advanced Studies), Tsukuba 305-0801, Japan

\section{J. Noaki}

High Energy Accelerator Research Organization (KEK), Tsukuba 305-0801, Japan

\begin{abstract}
We calculate the spectral function of the Mobius domain-wall Dirac operator utilizing a stochastic eigenvalue counting technique. From the low-end of the spectrum we extract the chiral condensate in 2+1-flavor QCD, and take the chiral and continuum limits. Lattice ensembles are those generated with Mobius domain-wall fermions at $a \sim 0.080,0.055$ and $0.044 \mathrm{fm}$.
\end{abstract}

34th annual International Symposium on Lattice Field Theory

24 -30 July 2016

University of Southampton, UK

${ }^{*}$ Speaker. 


\section{Introduction}

Vacuum expectation value of the scalar operator $\Sigma=-\langle\bar{q} q\rangle$ plays a role of an order parameter of spontaneous chiral symmetry breaking in QCD. It is related to the the eigenvalue density (or spectral function) of the Dirac operator

$$
\rho(\lambda)=\frac{1}{V}\left\langle\sum_{i} \delta\left(\lambda-\lambda_{i}\right)\right\rangle
$$

through the Banks-Casher relation $\rho(0)=\Sigma / \pi$ [1], which is valid in the thermodynamic limit, i.e. massless quark limit after taking the infinite volume limit. In our previous work using the overlap fermion formulation $[2,3]$ we calculated the low-lying eigenvalues of the Dirac operator on lattice ensembles at several quark masses. Analyzing the lattice data using the analytic formulae calculated within one-loop chiral perturbation theory in the $p$-regime and $\varepsilon$-regime [4], we extracted the chiral condensate, though the uncertainty due to finite lattice spacing and finite lattice volume was yet unexplored.

In this work we improve our previous calculation in several ways. By employing the Möbius domain-wall fermion instead of the overlap fermion, we are able to reduce the numerical cost and thus to extend the range of lattice parameters. In total, we analyze 15 lattice ensembles, with which the lattice cutoff covers $2.45,3.61$ and $4.50 \mathrm{GeV}$ while keeping the physical lattice extent of $2.7 \mathrm{fm}$. (Lattice sizes in actual simulation are $32^{3} \times 64,48^{3} \times 96$ and $64^{3} \times 128$.) Quark masses correspond to pion masses of 500, 400, 300 and $230 \mathrm{MeV}$. With the Möbius domain-wall fermion of finite fifth direction, the chiral symmetry is slightly violated and a small residual quark mass emerges. The size of residual mass observed in this work is order of $1 \mathrm{MeV}$ at most and much smaller on fine lattices. In this analysis such a small violation can be safely neglected. Each ensemble has sufficiently large statistics with 5,000 molecular dynamics time units. Details of the lattice parameters are in [5], where a full description of this work is available. The same set of lattice ensembles is also used for calculations of other physical quantities such as the $\eta^{\prime}$ meson mass [6], charm quark mass [7], short-distance current correlator [8, 9], as well as heavy-light meson decay constants [10] and semileptonic decay form factors [11].

\section{Eigenvalue filtering technique}

Explicit calculation of individual low-lying Dirac eigenvalues becomes increasingly more costly on larger volume lattices, since the number of eigenvalues needed to be calculated scales as the four-dimensional volume $V$. The corresponding computational cost thus grows as $V^{2}$. Instead, we utilize a stochastic method to estimate the number of eigenvalues in a given interval. The filtering function to achieve this can be constructed using a Chebyshev polynomial. The computational cost increases only as $V$, albeit some (controlled) approximation is introduced.

A similar method was first implemented in [12], where the number of eigenvalues below some threshold was calculated using a filtering function constructed with the Chebyshev polynomial and a rational function. The method introduced in our work is more flexible in a sense that the interval of eigenvalues can be arbitrarily chosen after the main calculation is done, and the spectrum in the whole range is obtained at once. A similar method has also been used in [14]. 
The method of stochastic counting of the eigenvalues can be summarized as follows. One tries to evaluate the number of eigenvalues of a hermitian matrix $A$ falling in an interval $[a, b]$ using $N_{v}$ (normalized) Gaussian random vectors $\xi_{k}$ :

$$
n[a, b]=\frac{1}{N_{v}} \sum_{k=1}^{N_{v}} \xi_{k}^{\dagger} h(A) \xi_{k} .
$$

Here, $h(A)$ is the filtering function that gives 1 in the range $[a, b]$ and 0 elsewhere. One can use the Chebyshev polynomial $T_{j}(x)$ to approximate $h(x)$ :

$$
h(x)=\sum_{j=0}^{p} g_{j}^{p} \gamma_{j} T_{j}(x)
$$

The coefficients $\gamma_{j}$ and $g_{j}^{p}$ are uniquely determined for a given $[a, b]$ within $[-1,+1]$ [13]. The conventional Chebyshev minmax approximation is obtained with $\gamma_{j}$, while the Jackson stabilization factor $g_{j}^{p}$ is introduced to suppress the oscillation typical in the Chebyshev expansion [13]. The Chebyshev polynomial $T_{j}(x)$ can be constructed using the recurrence formula

$$
T_{0}(x)=1, \quad T_{1}(x)=x, \quad T_{j}(x)=2 x T_{j-1}(x)-T_{j-2}(x) .
$$

Larger the order of polynomial $p$, the approximation is better. In our calculation we typically take $p=O(10,000)$, and the systematic error due to the approximation is about $1 \%$ for the smallest interval of the eigenvalues we analyze.

Therefore the recipe is as simple as follows.

1. Generate the Gaussian random noise vectors $\xi_{k}$.

2. Recursively calculate $T_{j}(A) \xi_{k}$ for $j=1, \ldots, p$.

3. Calculate inner-products $\xi_{k}^{\dagger} T_{j}(A) \xi_{k}$ and store them.

Then, the remaining analysis is off-line. One may first choose the interval $[a, b]$ and generate the corresponding coefficients $g_{j}^{p} \gamma_{j}$. The estimate of the mode number is given by

$$
\bar{n}[a, b]=\frac{1}{N_{v}} \sum_{k=1}^{N_{v}}\left[\sum_{j=0}^{p} g_{j}^{p} \gamma_{j}\left\langle\xi_{k}^{\dagger} T_{j}(A) \xi_{k}\right\rangle\right]
$$

after an ensemble average. Details of the method are found in [13].

For the Möbius domain-wall fermion, we calculate the eigenvalue density of the four-dimensional effective operator, which is constructed as

$$
D^{(4)}=\left[P^{-1}\left(D^{(5)}(m=1)\right)^{-1} D^{(5)}(m=0) P\right]_{11},
$$

where $D^{(5)}(m)$ represents the five-dimensional (5D) Möbius domain-wall operator with mass $m$. It is combined with the Pauli-Villas operator $(m=1)$ and the 4D surface (represented by the subscript "11") is taken after appropriate projection $P$ to define the 4D effective operator. (See, for instance, [15] for more details.) This 4D effective operator satisfies the Ginsparg-Wilson relation $D^{(4)} \gamma_{5}+$ 


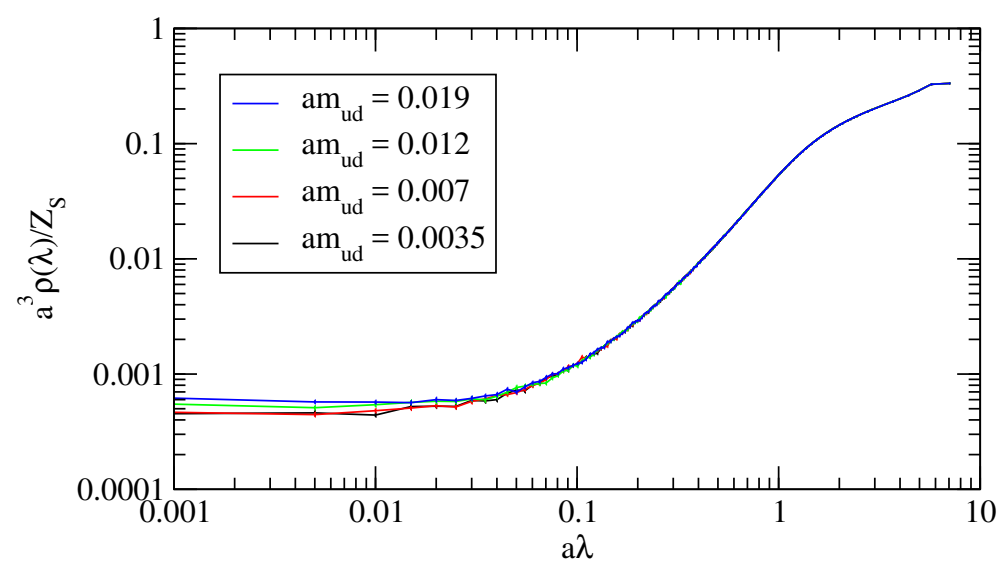

Figure 1: Spectral function in a logarithmic scale. The lattice data at $\beta=4.17(1 / a \sim 2.45 \mathrm{GeV})$ on $32^{3} \times 64$ lattices are plotted for four values of up and down quark masses.

$\gamma_{5} D^{(4)}=2 a D^{(4)} \gamma_{5} D^{(4)}$ to a good approximation. The eigenvalues of a hermitian operator $D^{(4) \dagger} D^{(4)}$ are in the range $[0,1]$. The eigenvalues of $D^{(4)}$ and those of $D^{(4) \dagger} D^{(4)}$ have an one-to-one mapping (up to a sign), and we can convert the spectrum of $D^{(4) \dagger} D^{(4)}$ to that of $D^{(4)}$. We then project the eigenvalue of $D^{(4)}$ to the imaginary axis.

An example of the entire spectrum thus obtained is shown in Figure 1, where the lattice data for the spectrum $\rho(\lambda)$ are plotted in a logarithmic scale. One can see that the spectrum increases rapidly toward higher eigenvalues, as expected. For near-zero eigenvalues $a \lambda \sim 0$, one can see some difference among different values of sea quark mass $a m_{u d}=0.0035-0.019$, while the high modes are nearly identical.

\section{Lattice results and chiral fits}

We calculate the spectral function on all ensembles available, with three lattice spacings and several sea quark masses of $2+1$ flavors.

We mainly analyze the lattice data near $\lambda \approx 0$ using the chiral perturbation theory formula calculated at the next-to-leading order [4]. That is written as

$$
\rho(\lambda)=\frac{\Sigma}{\pi}\left[1-\frac{1}{F^{2}}\left(\sum_{i} \operatorname{Re} \Delta\left(0, M_{v i}^{2}\right)-\operatorname{Re} G\left(0, M_{v v}^{2}, M_{v v}^{2}\right)-16 L_{6} \sum_{i} M_{i i}^{2}\right)\right]_{m_{v}=i \lambda} .
$$

The leading order low-energy constants, chiral condensate $\Sigma$ and pion decay constant $F$, are defined in the chiral limit. One of the low-energy constants, $L_{6}$, also appears at this order. The functions $\Delta\left(0, M^{2}\right)$ and $G\left(0, M^{2}, M^{2}\right)$ are

$$
\begin{aligned}
\Delta\left(0, M^{2}\right) & =\frac{M^{2}}{16 \pi^{2}} \ln \frac{M^{2}}{\mu_{s u b}^{2}}+g_{1}\left(M^{2}\right), \\
G\left(0, M^{2}, M^{2}\right) & =\frac{1}{2}\left[\Delta\left(0, M^{2}\right)+\left(M^{2}-M_{\pi}^{2}\right) \partial_{M^{2}} \Delta\left(0, M^{2}\right)\right] .
\end{aligned}
$$

They are evaluated at a "pion mass" as determined by the GMOR relation $M_{i j}^{2}=\left(m_{i}+m_{j}\right) \Sigma / F^{2}$, where the indices $i$ and $j$ label the sea quark mass or a fictitious valence quark mass $v$ taken at an 


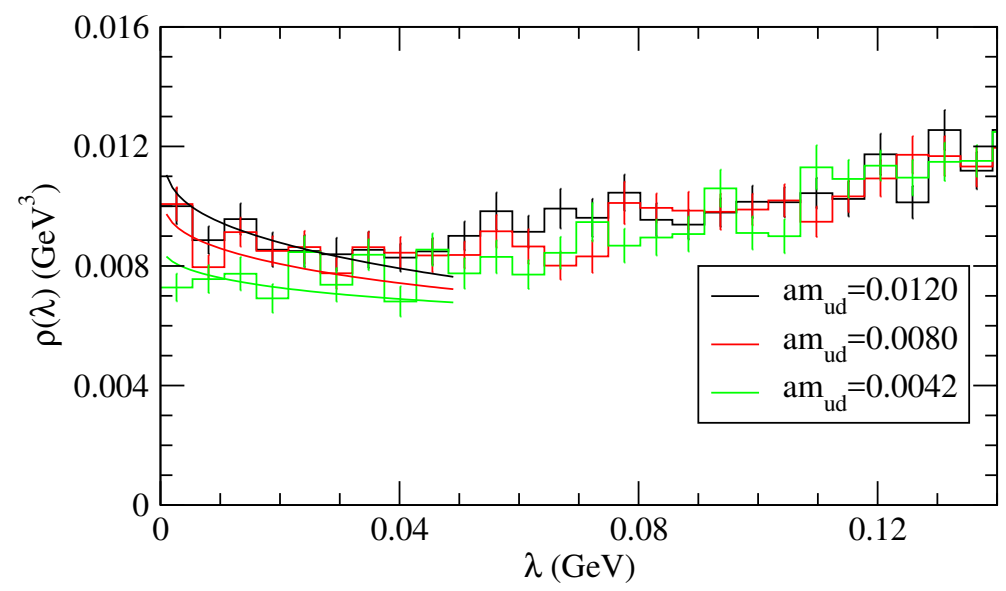

Figure 2: An example of the lattice data for $\rho(\lambda)$ plotted as a function of $\lambda$ in the physical unit $(\mathrm{GeV})$. Histograms are the lattice data at $\beta=4.35(1 / a \simeq 3.61 \mathrm{GeV})$ with three different values of sea quark mass. The results of the chiral and continuum fit (3.4) are also shown by lines.

imaginary value $m_{v}=i \lambda$. The last term in (3.2), i.e. $g_{1}\left(M^{2}\right)$, represents the finite volume effect, but it turned out to be negligible in our analysis. The scale parameter $\mu_{\text {sub }}$ denotes the renormalization scale taken at the $\rho$ meson mass.

We also introduce the terms that represent the discretization effect of $O\left(a^{2}\right)$ and the dependence on the strange quark mass $m_{s}$. They are assumed to be independent of other parameters and their effect is multiplied to $\rho(\lambda)$ as an overall factor:

$$
\left(1+c_{a} a^{2}\right)\left(1+c_{s}\left(M_{s s}^{2}-M_{s s}^{(\text {phys }) 2}\right)\right) \times \rho(\lambda) .
$$

The dependence on the strange quark mass is represented by that on the (fictitious) pseudo-scalar meson mass $M_{s s}$, and its physical value is taken with the GMOR relation from the experimental data.

Figure 2 shows an example of the lattice data for the ensembles of the middle lattice spacing $(1 / a \simeq 3.61 \mathrm{GeV})$. The spectral density observed on the lattice are multiplied by the renormalization factor $Z_{S}(2 \mathrm{GeV})$ to convert the results to those definition in the $\overline{\mathrm{MS}}$ scheme. The renormalization factors are independently calculated in [8].

In Figure 2 one can clearly observe an increase of the spectrum toward $\lambda=0$, which is consistent with the behavior predicted by chiral perturbation theory. We emphasize that this increase is a parameter-free prediction as one can see from (3.1), where there is no free parameter to control the $\lambda$ dependence and that is given by the functions $\Delta\left(0, M^{2}\right)$ and $G\left(0, M^{2}, M^{2}\right)$. It means that the spectral function reflects the effect of dynamical quarks in a way that the chiral effective theory predicts.

We also observe a quark mass dependence of the low-mode spectrum. We fit the lattice data using the formula (3.1), and the quark mass dependence determines the low-energy constant $L_{6}$.

We find that the results are nearly independent of the lattice spacing. In fact the fit yields the relevant parameter being zero: $c_{a}=0.00(15) \mathrm{GeV}^{2}$.

Finite volume effect is also invisible. Figure 3 shows the lattice data obtained at the same parameters other than the lattice volume, $32^{3} \times 64$ and $48^{3} \times 96$. The physical size of these lattices 


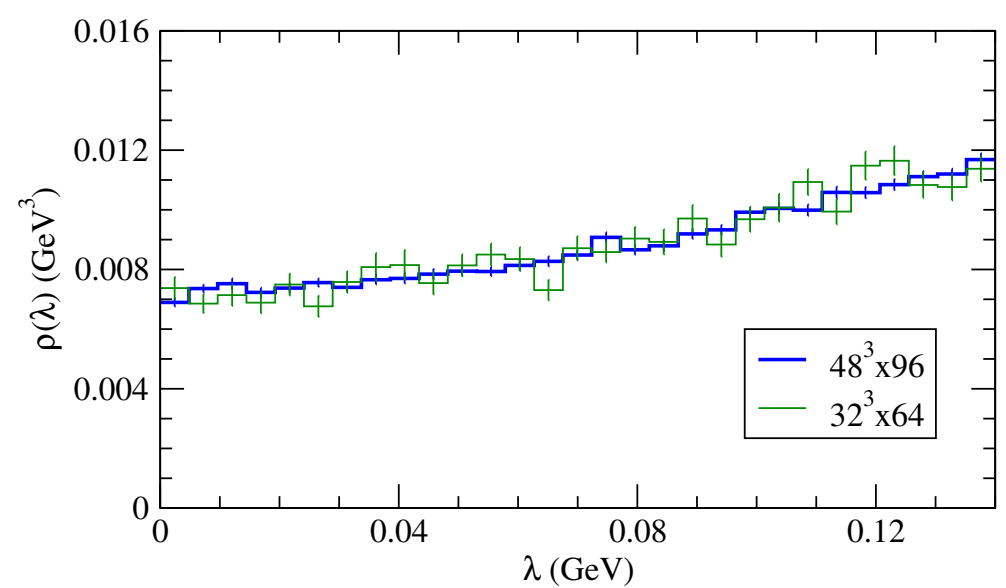

Figure 3: Spectral function calculated on two different volumes, $32^{3} \times 64$ and $48^{3} \times 96$. Data at $\beta=4.17$ $(1 / a \simeq 2.45 \mathrm{GeV})$ and pion mass about $230 \mathrm{MeV}$.

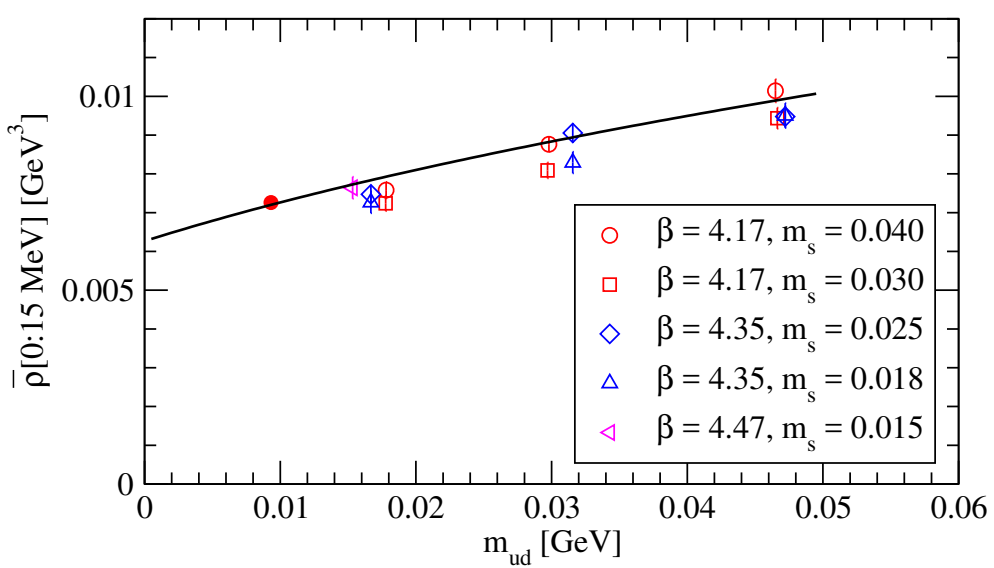

Figure 4: Chiral extrapolation of the partially integrated spectral function $\rho[0, \delta]$ with $\delta=15 \mathrm{MeV}$. The one-loop $\chi$ PT curve at $N_{f}=2$ is shown together with the lattice data (open symbols) at different lattice spacings and strange quark masses.

is about $2.6 \mathrm{fm}$ and $3.9 \mathrm{fm}$, respectively. The data are consistent with each other within the statistical error. The statistical error is smaller on the larger volume lattice because the number of eigenvalues in each bin is proportional to the volume.

Our fit result is

$$
\Sigma^{1 / 3}(2 \mathrm{GeV})=270.0(1.3)(1.3)(4.6) \mathrm{MeV},
$$

where the uncertainties are those of statistical error, the renormalization constant and the lattice scale. Adding in quadrature, the total error is $4.9 \mathrm{MeV}$, which is $1.8 \%$. The low-energy constant $L_{6}$ is also determined as $L_{6}=0.00016(6)$.

\section{Summary}

To summarize, the Chebyshev filtering technique allows precise evaluation of the eigenvalue density. The method is simple and flexible at the same time. 
The lattice data for the Dirac spectrum obtained on the ensembles generated with the Möbius domain-wall fermion at three lattice spacings are analyzed using the one-loop chiral perturbation theory formula. The lattice data are fully compatible with the effective theory and yield a precise determination of the chiral condensate, one of the most fundamental quantities to characterize the QCD vacuum.

We are grateful to Julius Kuti for private communications on the technique employed in this work. Numerical calculation was performed on the Blue Gene/Q supercomputer at High Energy Accelerator Research Organization (KEK) under a support of its Large Scale Simulation Program (No. 15/16-09). The code set Iroiro++ [16], which is highly optimized for Blue Gene/Q, is used. This work is supported in part by JSPS KAKENHI Grant Number 25800147, 26247043, 26400259 and by the Post-K supercomputer project through JICFuS.

\section{References}

[1] T. Banks and A. Casher, Nucl. Phys. B 169, 103 (1980). doi:10.1016/0550-3213(80)90255-2

[2] H. Fukaya et al. [JLQCD Collaboration], Phys. Rev. Lett. 104, 122002 (2010) [Phys. Rev. Lett. 105, 159901 (2010)] doi:10.1103/PhysRevLett.104.122002, 10.1103/PhysRevLett.105.159901 [arXiv:0911.5555 [hep-lat]].

[3] H. Fukaya et al. [JLQCD and TWQCD Collaborations], Phys. Rev. D 83, 074501 (2011) doi:10.1103/PhysRevD.83.074501 [arXiv:1012.4052 [hep-lat]].

[4] P. H. Damgaard and H. Fukaya, JHEP 0901, 052 (2009). doi:10.1088/1126-6708/2009/01/052, [arXiv:0812.2797 [hep-lat]].

[5] G. Cossu, H. Fukaya, S. Hashimoto, T. Kaneko and J. I. Noaki, Prog. Theor. Exp. Phys. (2016) 093B06. doi:10.1093/ptep/ptw129, arXiv:1607.01099 [hep-lat].

[6] H. Fukaya et al. [JLQCD Collaboration], Phys. Rev. D 92, no. 11, 111501 (2015) doi:10.1103/PhysRevD.92.111501 [arXiv:1509.00944 [hep-lat]].

[7] K. Nakayama, B. Fahy and S. Hashimoto, Phys. Rev. D 94, no. 5, 054507 (2016) doi:10.1103/PhysRevD.94.054507 [arXiv:1606.01002 [hep-lat]].

[8] M. Tomii et al. [JLQCD Collaboration], Phys. Rev. D 94, no. 5, 054504 (2016) doi:10.1103/PhysRevD.94.054504 [arXiv:1604.08702 [hep-lat]].

[9] M. Tomii et al., PoS(LATTICE2016)289.

[10] B. Fahy et al., PoS(LATTICE2016)118.

[11] T. Kaneko et al., PoS(LATTICE2016)297.

[12] L. Giusti and M. Luscher, JHEP 0903, 013 (2009) doi:10.1088/1126-6708/2009/03/013 [arXiv:0812.3638 [hep-lat]].

[13] E. Di Napoli, E. Polizzi, Y. Saad, arXiv:1308.4275 [cs.NA].

[14] Z. Fodor, K. Holland, J. Kuti, S. Mondal, D. Nogradi and C. H. Wong, PoS LATTICE 2015, 310 (2016) [arXiv:1605.08091 [hep-lat]].

[15] P. A. Boyle [UKQCD Collaboration], PoS LATTICE 2014, 087 (2015).

[16] G. Cossu, J. Noaki, S. Hashimoto, T. Kaneko, H. Fukaya, P. A. Boyle and J. Doi, arXiv:1311.0084 [hep-lat]. 